\title{
CRASHWORTHINESS IMPROVEMENT OF HEXAGONAL TUBULAR STRUCTURES BY USING TAILOR WELDED BLANKS
}

\author{
CIUBOTARIU, V[lad] A[ndrei]
}

\begin{abstract}
This paper investigates the energy absorption characteristics of hexagonal thin-walled structures under quasistatic / dynamic axial compression. These structures are made from homogenous steel blanks and tailor welded blanks (TWB). The fabrication procedure includes a plastic forming process in which the edges of the blanks are firmly held in place. The distribution of plastic strain, thickness variation and strain hardening within the materials are strongly influencing the collapsing mode of the structures. From the experimental results, it was found that by using TWB hexagonal structures the specific energy absorption can be improved by up to $17 \%$, and peak force can be increased by up to $32 \%$ in the case of quasistatic loadings; in the case of dynamic loading the specific energy absorption can be improved by up to $12 \%$ and peak force can be increased by up to $14 \%$. The influence of several parameters, including the formability characteristics of the materials within the tailor welded blanks and the features of the collapse modes are described and analyzed. The use of tailor welded blanks structures is found to be an effective way to improve the crashworthiness of tubular structures.

Keywords: hexagonal tube, thin-walled structure, energy absorption, crashworthiness, TWB, collapse mode
\end{abstract}

\section{INTRODUCTION}

In the recent years, the number of vehicles has rapidly increased due to new developed technologies in the field of transportation systems. In fact, these increase caused the occurrence of more and more vehicle crash accidents which emphasized a new problem: active and passive protection of vehicles.

Numerous types of materials and structures with excellent energy absorption qualities have been developed and investigated to dissipate energy during an impact event and hence protect the structure in a controlled manor.

High strength, low weight precision tube solutions have already been developed for body-in-white (BIW) applications such as door beams and the crash management system (fig.1). Tubular products are also used in suspension systems where they form twist beams, trailing arms, shock absorbers and the engine cradle. Inside the vehicle you will find tubes in seat frames, airbags and the steering system [1].

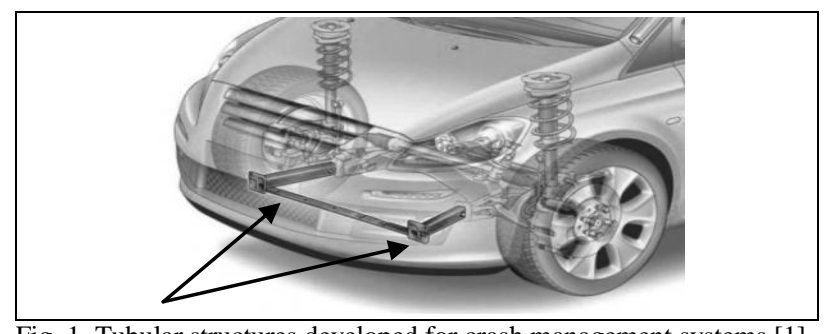

Fig. 1. Tubular structures developed for crash management systems [1]
The structures considered to have good energy absorption behavior used in the majority of transportation vehicles are the thin-walled (tubular) structures. These types of structures are fabricated from different ductile materials and used frequently as energy absorbers in various crashworthiness applications, especially in automotive industry to mainly protect the occupants and cargo. For instance, during a frontal car crash, one of the most common impact situations, the front rails represent the main deformation component designed to dissipate the kinetic energy in a stabile and controlled manor [2].

Tubular structures can be designed with different geometries and shapes like: cylindrical, rectangular, square or honeycomb. These types of structures are known as excellent deformable elements due to their capacity to deform progressively and stably [3].

According to previous studies, tubular structures can collapse in some different deformation modes as it follows: axisymmetric mode (fig.2), known as ring or concertina mode, non-symmetric mode, also known as diamond or mixed mode. The deformation mode a tubular structure collapses into largely depends on the geometry of the structure. The kinetic energy will be absorbed trough the progressive buckling of the structure occurred during the collapse [4].

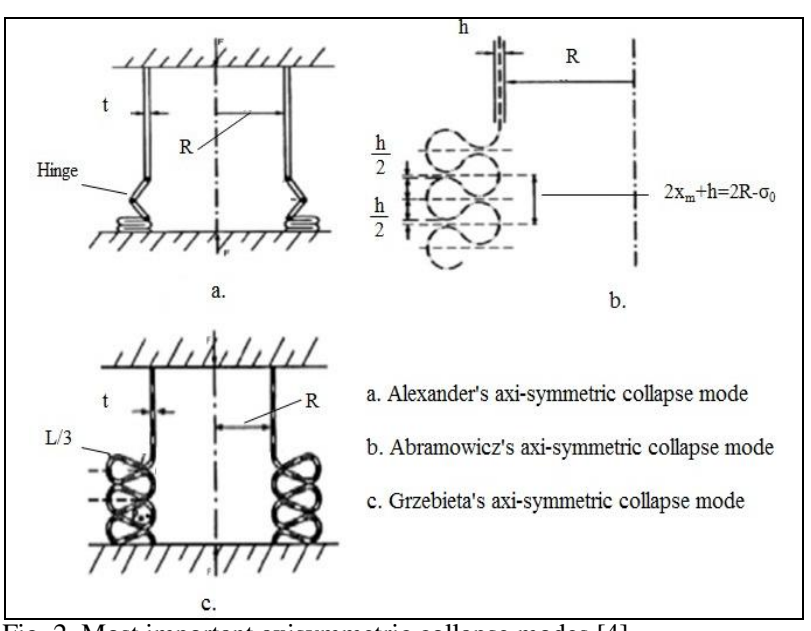

Fig. 2. Most important axisymmetric collapse modes [4]

Also, the materials used to fabricate these structures are as important as the structure itself. Usually, both light and resistant materials are used. Some of these materials can be HSLA (High Strength Low Alloy) steel, UHSLA (Ultra High Strength Low Alloy) steel or even aluminum. It is known that tubular structures can be either empty or filled with ultra light foam like materials such as polyurethane foam or aluminum foam. In the fabrication 
process of the structure itself, homogenous materials are often used but an attractive solution for the automotive companies is materialized by the use of tailor welded blanks (TWB).

A TWB is a sheet metal that combines several grades and/or various thickness and/or different coatings, the different parts being laser welded together in order to place the best material at the best place in the right thickness for the real "tailor made" solution after the forming process. TWB are especially in use in the automotive industry, for the BIW and the closure of a car. Using TWB ensures the designers of some advantages which are very hard to ignore nowadays such as [6]:

- Reduction of the cars global weight by optimizing the material use;

- Increase of the technical performances without increasing the weight by having a higher stiffness and better energy absorption;

- Simplification of the production process by reducing the number of parts and forming or assembly tools needed.

To understand the mechanism of the crashworthiness improvement for tubular structures made from TWB, experimental analysis regarding quasistatic / dynamic axial loading of hexagonal structures was carried out in this study.

\section{EXPERIMENTAL CONDITIONS}

\subsection{Materials and formability characterization}

In this study homogenous and LASER welded samples were used. Both the homogenous (with parent materials) and TWB samples were obtained using two steel marks as it follows: FeP-05-MB, which in this study will be referred to as STEEL 1, and SPE-220-BH, which in this study will be referred to as STEEL 2. These two materials are different regarding both mechanical characteristics and thickness respectively. The thickness of the sheet metals was $0.75 \mathrm{~mm}$ for STEEL 1 and 0.7 $\mathrm{mm}$ for STEEL 2. The mechanical characteristics of both steel marks used in the present study are shown in tab.1.

\begin{tabular}{|l|c|c|c|c|}
\cline { 2 - 5 } \multicolumn{1}{c|}{} & u.m. & $\begin{array}{c}\text { STEEL } \\
1\end{array}$ & $\begin{array}{c}\text { STEEL } \\
2\end{array}$ & TWB \\
\hline Young modulus & {$[\mathrm{MPa}]$} & 163174 & 204175 & 211995 \\
\hline Yield point & {$[\mathrm{MPa}]$} & 140.46 & 191.43 & 230.35 \\
\hline Tensile strength & {$[\mathrm{MPa}]$} & 370.95 & 378.47 & 392.88 \\
\hline Total elongation & {$[\%]$} & 37.22 & 26.51 & 25.82 \\
\hline Plastic strain ratio, $r$ & - & 1.79 & 2.21 & 2.69 \\
\hline $\begin{array}{l}\text { Coefficient of } \\
\text { resistance, } K\end{array}$ & - & 490.6 & 502.9 & 514 \\
\hline $\begin{array}{l}\text { Strain hardening } \\
\text { exponent, } n\end{array}$ & - & 0.25 & 0.19 & 0.17 \\
\hline
\end{tabular}

Tab. 1. Mechanical characteristics of both steel marks and TWB used in the present study

The formability characterization of both homogenous blanks and TWB was obtained by Marciniak formability test. Using the data from the tests and the modified Hollomon's law of plasticity (1) the forming limit curves of the studied materials were obtained. These curves are presented in fig. 3 .

$$
\sigma=\left[1+\left(\frac{\dot{\varepsilon}}{C}\right)^{\frac{1}{P}}\right] K\left(\varepsilon_{e}+\varepsilon_{p}^{e f}\right)^{n}
$$

where: $\dot{\varepsilon}$ is the strain rate, $C$ and $P$ are the CowperSymonds parameters regarding the strain rate, $K$ is the resistance coefficient, $\varepsilon_{e}$ represents the elastic deformation, $\varepsilon_{p}{ }^{e f}$ is the real (effective) plastic deformation and $n$ represents the strain hardening coefficient. In order to obtain the forming limit curves of the studied materials, some other parameters were used, as if follows: density of the materials $-\rho$, Young's modulus $-E$, Poisson's coefficient $-v$.

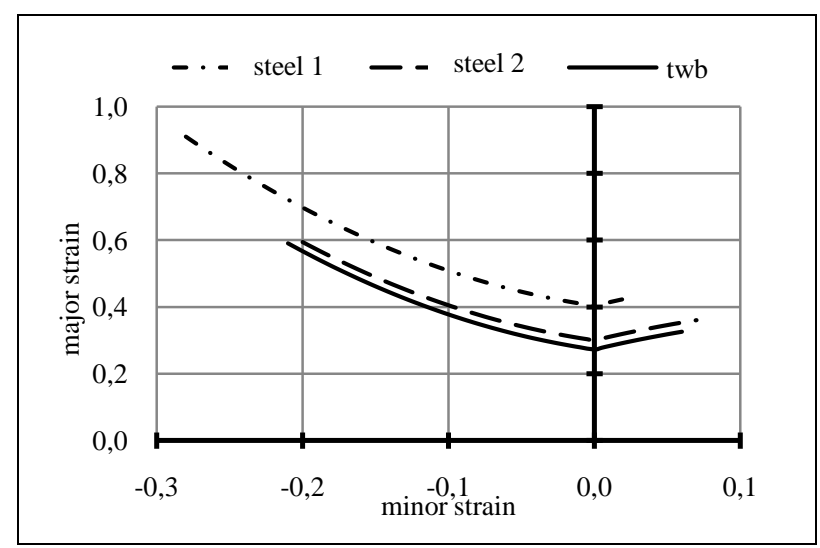

Fig. 3. Forming limit curve of the parent materials and the TWB [4]

The forming limit curves obtained after data processing and presented above are named by the scientific community as the instability forming limit curves [5]. As shown in fig.3, the TWB assembly presents lower formability capacities compared to the parent materials. This behavior is strongly influenced by the weld line which presents the highest coefficient of resistance.

\subsection{Experimental and analytical procedure}

In order to determine the behavior regarding the tubular structures under axial loading, two different crashing conditions were considered: quasistatic and dynamic loading. A hydraulic press was used to conduct the quasistatic loading tests and gravitational hammer type equipment (height $=3150 \mathrm{~mm}$ and load $=110 \mathrm{~kg}$ ) was used to conduct the dynamic loading tests. To measure the quasistatic / dynamic loads undertaken by the samples, a class 5 Kaliber force transducer was used. Also, the data acquisition was conducted through a $600 \mathrm{~Hz}$ Spider 8 acquisition system and Catman EXPRESS software.

The tubular structures were subsequently obtained by plastic forming rectangular sheet metals as it is shown in fig.4. Using a modified polymer adhesive solution for the formed parts, the tubular structures were fabricated. The specific height of the structures was established to be 200 $\mathrm{mm}$ and the width of $100 \mathrm{~mm}$.

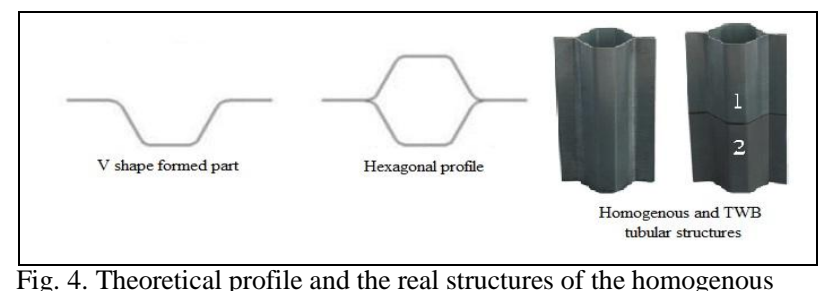
and TWB tubular structures 
In order to properly characterize these structures the following parameters were used: $F_{\max }-$ maximum force needed to deform the structure, $E_{a}$ - absorbed energy (2), $F_{m}$ - medium force of deformation (3), $A_{e s}$ - specific energy absorption (4), $E_{F}$ - deformation force efficiency (5). The mathematical expressions of these parameters are presented as it follows:

$$
E_{a}=\int_{0}^{d_{\max }} F \delta d \delta
$$

Where: $F$ is the force needed to deform the structure, $d$ is the instantaneous distance and $d_{\max }$ is the maximum deformation distance.

$$
F_{m}=\frac{1}{d} \int_{0}^{d_{\max }} F \delta d \delta=\frac{E_{t}}{d_{t}}
$$

Where: $E_{t}$ represents the total absorbed energy and $d_{t}$ is the overall distance of deformation.

$$
A_{e s}=\frac{E_{a}}{m}
$$

Where: $m$ represents the total mass of the tubular structure.

$$
E_{F}=\frac{F_{m}}{F_{\max }}
$$

Where: $F_{\max }$ is the maximum force needed to deform the structures.

\section{EXPERIMENTAL RESULTS AND DISCUTIONS}

The samples obtained under the quasistatic loading present a more stable and symmetrical bucking mode comparing to the samples obtained after dynamic loading. Some examples of such crashed structures under quasistatic and dynamic loading are presented in fig.5.

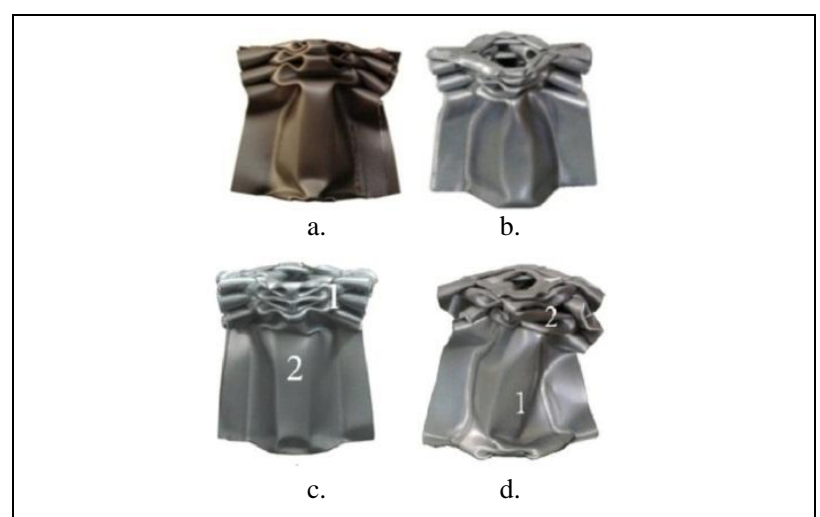

Fig. 5. Deformed structures with different type of fabrication mode and different type of loading: a) homogenous structure deformed under quasistatic loading, b) homogenous structure deformed under dynamic loading, c) TWB structure deformed under quasistatic loading, d) TWB structure deformed under dynamic loading

The diagrams regarding the force and absorbed energy in conjunction with displacement corresponding to homogenous and TWB structures are presented in fig. 6 and fig 7. The continuous line corresponds to the TWB hexagonal structures and regarding the force / displacement part of the diagram it presents itself to have a regulate path in both quasistatic and dynamic loading tests. According to these measurements and graphic representations, these structures have the best energy absorbing capacity comparing to the structures made from homogenous sheet metals.

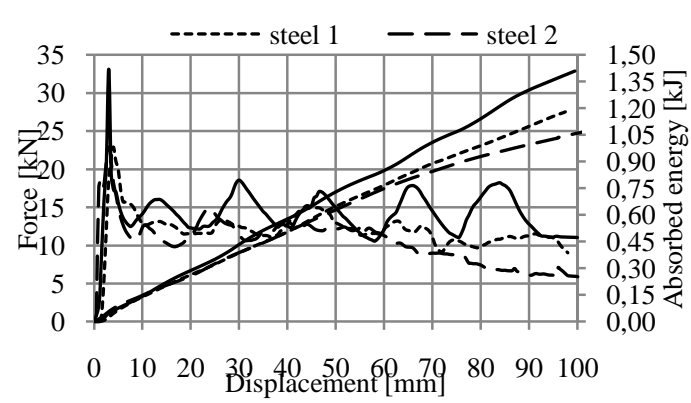

Fig. 6. Force and absorbed energy in conjunction with displacement corresponding to homogenous and TWB structures in the case of quasistatic loading

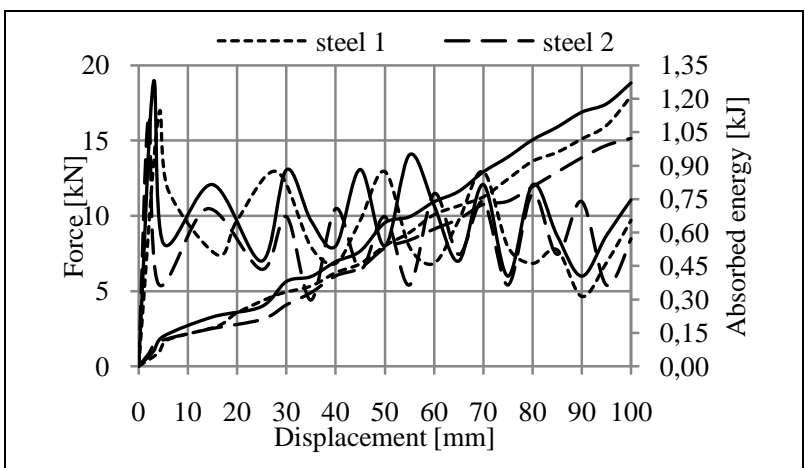

Fig.7. Force and absorbed energy in conjunction with displacemen corresponding to homogenous and TWB structures in the case of dynamic loading

Furthermore, the TWB structures deformed under quasistatic loading presents a very strait and stabile buckling mode. The numerical characterization of the parameters involved in this study is presented in tab.2 and tab. 3 respectively.

\begin{tabular}{|c|c|c|c|}
\hline Parameter & Steel 1 & Steel 2 & TWB \\
\hline$F_{\max }[\mathrm{kN}]$ & 22.92 & 27.45 & 33.10 \\
\hline$E_{a}[\mathrm{~J}]$ & 1219.15 & 1108.98 & 1408.91 \\
\hline$F_{m}[\mathrm{kN}]$ & 12.19 & 11.09 & 14.09 \\
\hline$A_{e s}[\mathrm{~J} / \mathrm{gr}]$ & 4.01 & 3.91 & 4.63 \\
\hline$E_{F}$ & 0.53 & 0.40 & 0.43 \\
\hline
\end{tabular}

Tab. 2. Numerical characterization of the crash test parameters obtained under quasistatic loading

\begin{tabular}{|c|c|c|c|}
\hline Parameter & Steel 1 & Steel 2 & TWB \\
\hline$F_{\max }[\mathrm{kN}]$ & 16.58 & 16.64 & 18.87 \\
\hline$E_{a}[\mathrm{~J}]$ & 1211.64 & 1021.77 & 1270.17 \\
\hline$F_{m}[\mathrm{kN}]$ & 11.69 & 9.47 & 12.05 \\
\hline$A_{e s}[\mathrm{~J} / \mathrm{gr}]$ & 3.86 & 3.48 & 4.10 \\
\hline$E_{F}$ & 0.71 & 0.56 & 0.63 \\
\hline
\end{tabular}

Tab. 3. Numerical characterization of the crash test parameters obtained under dynamic loading

As the graphs in fig 8-9 show, the TWB structures present the best specific energy absorption related to the absorbed energy, meaning that these structures absorb more kinetic energy at lower weight. The slight difference between the structures made from STEEL 1 and the ones made from TWB, favoring the second ones indicate that STEEL 2 is very influenced by inertia effect. 


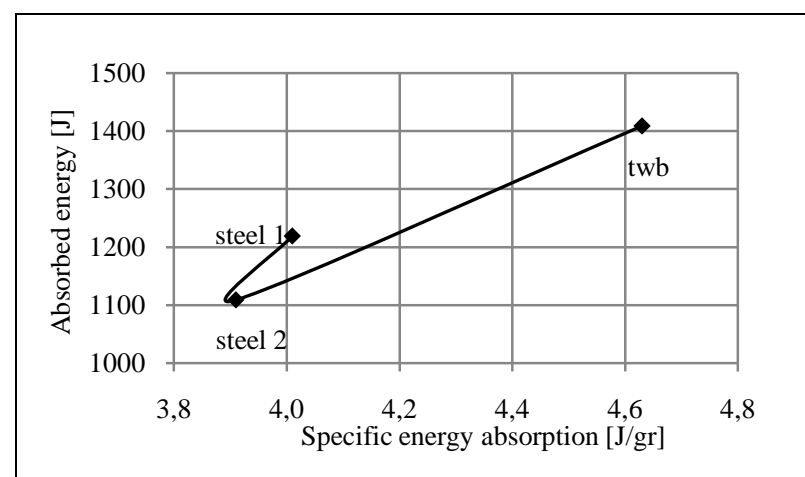

Fig. 8. Correlation between specific energy absorption and absorbed energy for homogenous and TWB structures under quasistatic loading

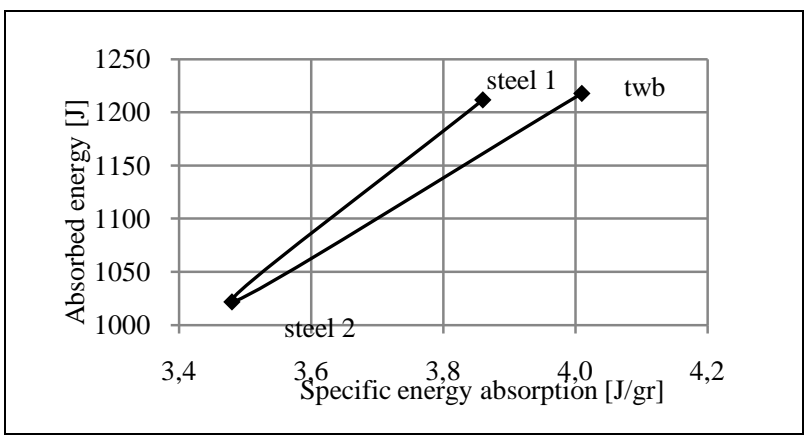

Fig. 9. Correlation between specific energy absorption and absorbed energy for homogenous and TWB structures under dynamic loading

Also it is indicated in the presented data that in the case of dynamic loadings the total absorbed energy has a decreased range of variation comparing to the quasistatic loading results. This may be also caused by the inertia effect over the materials, in which case it is normal that in quasistatic loadings the inertia effect to be toward null values.

For a better understanding of the inertia effect over these structures, a correlation between the absorbed energy and the strain hardening parameter of the homogenous structures was configured. As it is shown in fig.10, the decreased energy absorption capacity of STEEL 2 is related to the strain hardening coefficient, which its value is lower comparing to the other material.

Also, the inertia effect in conjunction with the thickness and strain hardening of STEEL 2 caused a serious decrease of the maximum force needed to initiate the deformation of the structures in the case of dynamic loading (fig.11). It is noticed that for dynamic loadings the force variation range between the homogenous and TWB structures is reduced.

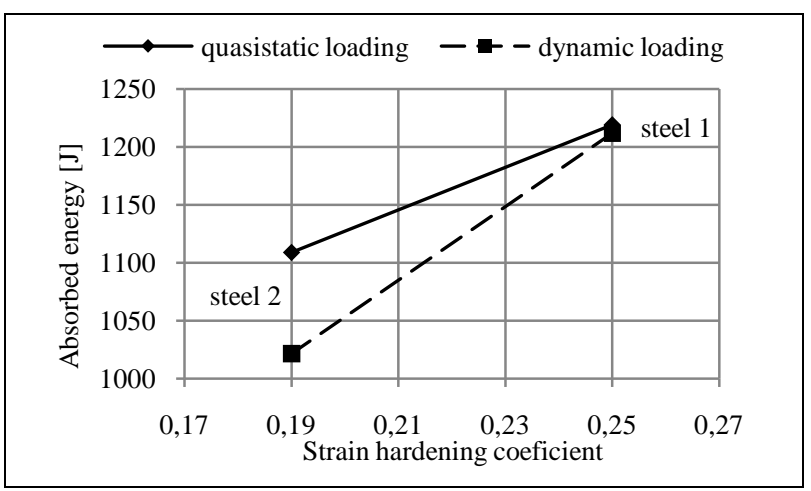

Fig. 10. Correlation between the strain hardening coefficient and the absorbed energy in both quasistatic and dynamic loading corresponding to homogenous structures

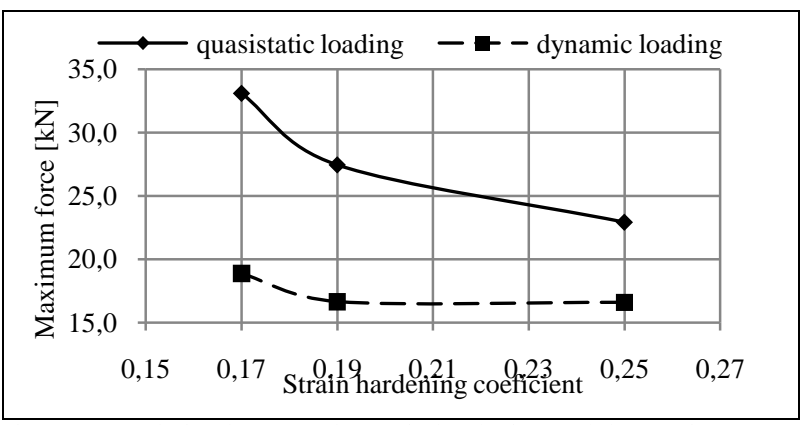

Fig. 11. Correlation between the strain hardening and the maximum force needed to initiate the deformation of the structures in both quasistatic and dynamic loading

\section{CONCLUSIONS}

From the experimental results, it was found that by using TWB hexagonal structures the specific energy absorption can be improved by up to $17 \%$, and peak force can be increased by up to $32 \%$ in the case of quasistatic loadings; in the case of dynamic loading the specific energy absorption can be improved by up to $12 \%$ and peak force can be increased by up to $14 \%$. The behavior of the hexagonal structures is critically influenced by materials that present a lower strain hardening characteristic which causes an important inertia effect, especially in the case of dynamic loadings.

In the case of TWB structures, the weld line plays an important role in the distribution of stresses along both materials during the crash tests. Therefore, the superior resistance of the weld line and its reduced dimensions makes the conjunction of the mechanical properties of both materials possible, conferring better energy absorption characteristics to the structures. Therefore, the use of tailor welded blanks structures is found to be an effective way to improve the crashworthiness of tubular structures

\section{ACKNOWLEDGEMENTS}

The author gratefully acknowledge the financial support from the Romanian Ministry of Education, Research, Youth and Sport through project TE 256/2010 and technical support of CCIMT Research Centre from "Vasile Alecsandri" University of Bacau - Romania

\section{REFERENCES}

[1] Pauwels P. (2011) AMC Update Client Magazine, pp.12, November 2011, Available from http://www.arcelormittal.com/ fce/repository/Update/EN_UpdateFCE_November11.pdf, Accessed: 2012-04-24

[2] Zaini A. (2009). Impact and energy absorption of empty and foam-filled conical tubes, Ph.D. thesis, Queensland University of Technology, Brisbane, Australia

[3] Hamza T.K. (2008). Design for vehicle structural crashworthiness via crash mode matching, Ph.D. thesis, University of Michigan, USA

[4] Ciubotariu V.A. (2011). Theoretical and experimental research regarding the influence of the weld line on the quality and resistance characteristics of the drawn parts made from tailor welded blanks, Ph.D. thesis, "Vasile Alecsandri" University of Bacau, Romania

[5] Marciniak Z., Duncan J. (2002) Mechanics of sheet metal forming: 2nd edition, Butterworth-Heinemann, ISBN 0750653000, London

[6] Zadpoor A.A., Sinke J., Benedictus R. (2007). Mechanics of TWB: An Overview, Key Engineering Material, Vol. 344, pp. 373-382 